\title{
Radiation exposure and mortality risk from CT and PET imaging of patients with malignant lymphoma
}

\author{
R. A. J. Nievelstein - H. M. E. Quarles van Ufford • \\ T. C. Kwee • M. B. Bierings • I. Ludwig • F. J. A. Beek • \\ J. M. H. de Klerk • W. P. Th. M. Mali • P. W. de Bruin • \\ J. Geleijns
}

Received: 31 October 2011 /Revised: 6 February 2012 / Accepted: 20 February 2012 / Published online: 27 April 2012

(C) The Author(s) 2012. This article is published with open access at Springerlink.com

\begin{abstract}
Objective To quantify radiation exposure and mortality risk from computed tomography (CT) and positron emission tomography (PET) imaging with ${ }^{18} \mathrm{~F}$-fluorodeoxyglucose $\left({ }^{18} \mathrm{~F}\right.$ FDG) in patients with malignant lymphoma (Hodgkin's disease [HD] or non-Hodgkin's lymphoma [NHL]).

Methods First, organ doses were assessed for a typical diagnostic work-up in children with HD and adults with NHL. Subsequently, life tables were constructed for assessment of radiation risks, also taking into account the disease-related mortality.

Results In children with HD, cumulative effective dose from medical imaging ranged from $66 \mathrm{mSv}$ (newborn) to $113 \mathrm{mSv}$ (15 years old). In adults with NHL the cumulative
\end{abstract}

H. M. E. Quarles van Ufford

Department of Radiology, Medical Center Haaglanden,

The Hague, The Netherlands

R. A. J. Nievelstein $(\varangle) \cdot$ T. C. Kwee · F. J. A. Beek $•$

W. P. T. M. Mali

Department of Radiology (E 01.132), University Medical Center,

P.O. Box 85500, 3508 CX Utrecht, The Netherlands

e-mail: r.a.j.nievelstein@umcutrecht.nl

\section{B. Bierings}

Department of Pediatric Hematology, University Medical Center,

Utrecht, The Netherlands

I. Ludwig

Department of Hematology, University Medical Center,

Utrecht, The Netherlands

J. M. H. de Klerk

Department of Nuclear Medicine, Meander Medical Center,

Amersfoort, The Netherlands

P. W. de Bruin · J. Geleijns

Department of Radiology, University Medical Center,

Leiden, The Netherlands effective dose from medical imaging was $97 \mathrm{mSv}$. Average fractions of radiation-induced deaths for children with HD [without correction for disease-related mortality in brackets] were $0.4 \%[0.6 \%]$ for boys and $0.7 \%$ [1.1\%] for girls, and for adults with NHL $0.07 \%$ [0.28\%] for men and $0.09 \%$ [0.37\%] for women.

Conclusion Taking into account the disease-related reduction in life expectancy of patients with malignant lymphoma results in a higher overall mortality but substantial lower incidence of radiation induced deaths. The modest radiation risk that results from imaging with CT and ${ }^{18} \mathrm{~F}$-FDG PET can be considered as justified, but imaging should be performed with care, especially in children.

Key Points

- Survival of malignant lymphoma has improved dramatically over the past decades.

- PET and CT currently play important roles for malignant lymphoma patients.

- The potential hazard of ionising radiation has become an increasingly important issue.

- When assessing radiation risks, disease-related reduction in life expectancy should be considered.

- CT and ${ }^{18}$ F-FDG PET create a modest radiation-induced mortality risk.

Keywords Radiation exposure - Medical imaging ·

Malignant lymphoma $\cdot$ Computed tomography .

Positron emission tomography

\section{Introduction}

Imaging plays an important role in the management of patients with malignant lymphoma (Hodgkin's disease [HD] and non-Hodgkin's lymphoma [NHL]). Accurate 
imaging is important for determining the stage of disease, which guides the treatment strategy and influences the prognosis [1]. Imaging is also important for assessing response to therapy and detecting tumour persistence or recurrence [2-4]. Computed tomography $(\mathrm{CT})$ and, more recently, positron emission tomography (PET) with ${ }^{18} \mathrm{~F}$-fluorodeoxyglucose $\left({ }^{18} \mathrm{~F}-\mathrm{FDG}\right)$ have become indispensable tools in oncological imaging [2, 5-9]. However, CT and ${ }^{18}$ F-FDG PET result in exposure of the patient to ionising radiation, which is associated with a carcinogenic risk [10-17].

There is a worldwide growing concern about radiation exposure in medical imaging [12, 18-21]. Estimates of the National Council on Radiation Protection and Measurements (NCRP) Scientific Committee in the United States in 2006 show an almost sixfold increase in the per capita dose from medical exposure to about $3 \mathrm{mSv}$ compared with 1982. CT and nuclear medicine examinations are the largest contributors [22].

Therapeutic advances have dramatically improved the survival of patients with malignant lymphoma over the past decades. HD can nowadays be cured in at least $80 \%$ of patients [23-25]. Therefore, current treatment strategies not only aim at maximising curative success but also at minimising (late) toxicity, such as infertility, premature menopause, cardiac disease, and most importantly, risk of second neoplasms [24, 25]. In this context, the potential hazard of ionising radiation that is associated with diagnostic CT and PET in patients with malignant lymphoma is an increasingly important issue. Therefore, performing dose and risk assessment should be prioritised for circumstances with high cumulative radiation exposure for individual patients and better survival rates. This is particularly true when patients are young, such as children with HD, and when the cumulative radiation dose from imaging is expected to fall within the range of 5-150 $\mathrm{mSv}$, such as during follow-up of patients treated for malignant lymphoma $[10-13,15]$. This latter dose range is based on the studies in the subgroup of atomic bomb survivors who received low doses of radiation, ranging from 5 to $150 \mathrm{mSv}$ (mean dose of $40 \mathrm{mSv}$ ), which suggested a significant increase in the overall risk of cancer in this subgroup [14-16].

The aim of this study was to perform accurate radiation risk assessment for imaging of patients with malignant lymphoma. To achieve our goal, the radiation exposure of CT and ${ }^{18}$ F-FDG PET was calculated for a typical work-up for children with HD in different age categories, and for adults with NHL. We expected that disease-related mortality would have a significant effect on the assessment of radiation risk in patients with malignant lymphoma, and that diseaserelated mortality would be much higher compared with radiation-induced mortality. This expectation is in accordance with a very recent publication by Brenner et al.
[26]. Advanced radiation risk assessment, based on the demographic methodology of life tables, was developed to take into account these effects.

\section{Materials and methods}

\section{Clinical practice of imaging}

Malignant lymphoma comprises a heterogeneous group of diseases, differing with regard to histology, treatment and outcome. It is beyond the scope of this study to encompass all the different entities. Instead we focus on the most common types of malignant lymphoma; i.e. HD in children and diffuse large B-cell lymphoma (DLBCL) in adults [27-30]. The most typical imaging strategy for children with HD (age $<18$ years) and for adults with DLBCL was used in this study. In children with HD, the imaging strategy was based on the protocol according to the Children's Oncology Group [COG]; in adults with DLBCL, the imaging strategy was based on the HOVON 84 international multicentre trial currently running in The Netherlands [www.hovon.nl, EudraCTnr. 2006-005174-42] (Table 1).

Imaging with ultrasound and chest radiography were not considered in this study, as ultrasound is not associated with radiation exposure, and the radiation exposure from chest radiography is negligible compared with $\mathrm{CT}$ and ${ }^{18} \mathrm{~F}-\mathrm{FDG}$ PET.

\section{Radiation exposure from CT}

There was no information available that allowed for assessment of organ doses in paediatric patients undergoing CT. Therefore we created Medical Internal Radiation Dose (MIRD) paediatric patient models in five general categories according to age and weight: newborn $(3.6 \mathrm{~kg}), 1$ year old $(9.7 \mathrm{~kg}), 5$ years old $(19.8 \mathrm{~kg}), 10$ years old $(33.2 \mathrm{~kg})$ and 15 years old $(56.8 \mathrm{~kg})$. The adult MIRD patient model represents an average-sized patient of $74 \mathrm{~kg}$. All these hermaphrodite patient models (MIRD V) are described by spheres, ellipsoids and cones, as illustrated in Fig. 1 [31]. For assessment of radiation exposure from $\mathrm{CT}$, we used these patient models in combination with an algorithm for Monte Carlo dose calculations (ImpactMC software, version 1.0, VAMP, Erlangen, Germany) [31, 32]. The Monte Carlo dose calculations are based on a virtual model of the CT system with respect to geometry, X-ray spectrum, filtration and $\mathrm{CT}$ parameters. The simulation is performed on $3 \mathrm{D}$ voxelised versions of the MIRD $\mathrm{V}$ phantoms. To each voxel in the volume during the simulation, a density value and a mass attenuation coefficient are assigned corresponding to five different materials: air, lung, soft tissue, fat and bone. During the simulation of a CT examination, the energy 
Table 1 The imaging strategy for children with Hodgkin's disease $(H D)$ and adults with diffuse large B-cell lymphoma $(D L B C L)$

\begin{tabular}{lll}
\hline & Children with HD & Adults with DLBCL \\
\hline Most common group & Intermediate Risk Strategy: stage I-A bulky disease, I-AE, IB, II-A bulky & Stage II-IV \\
Initial diagnostic & disease, II-AE, II-B, III-A, III-AE, III-AS, III-AE+S, IV-A en IV-AE & 1 CT of neck-chest-abdomen \\
& 1 CT of neck-chest-abdomen & 1 chest X-ray \\
& 1 whole-body ${ }^{18}$ F-FDG PET & 2 CT scans of neck-chest-abdomen \\
& 1 chest X-ray & 1 whole-body ${ }^{18}$ F-FDG PET scan \\
Therapeutic phase & 2 CTs of neck-chest-abdomen & \\
& 1 whole-body ${ }^{18}$ F-FDG PET (directly after therapy) & 4 CT scans of neck-chest-abdomen \\
& 2 chest X-rays & 2 chest X-rays \\
Follow-up & 2 CTs of neck-chest-abdomen & \\
& 7 CTs of neck and chest (for most common supradiaphragmatic & At $6,12,18$ and 24 months \\
& stage I and II disease) & chest X-rays \\
Timing of CT during follow-up & At $3,6,9,12,15,18,24,36$ and 60 months &
\end{tabular}

deposited in each voxel (absorbed dose) is accumulated and saved in an additional volume. The manufacturer of the Aquilion CT system (Toshiba Medical Systems) disclosed two measured X-ray spectra $(100 \mathrm{kV}$ and $120 \mathrm{kV})$ and information about the design of the CT system. This information was implemented in the Monte Carlo simulation. A MatLab script (The MathWorks, Natick, MA, USA) was used to extract organ doses from the calculated dose distributions within the MIRD mathematical phantoms.

In $\mathrm{CT}$ it is common practice to adjust acquisition parameters to the size of the patient, and to the clinical application [33]. The acquisition protocol we used was based on local and optimised practices, and was checked against general recommendations, particularly with regard to the optimisation of the paediatric acquisitions. We thus derived the following clinical CT acquisition parameters. For small children, a tube voltage of $100 \mathrm{kV}$ was used (weight $<30 \mathrm{~kg}$ ), and for larger children and adults a tube voltage of $120 \mathrm{kV}$ $(\geq 30 \mathrm{~kg}$ ), both in combination with a pitch factor of 0.83 . Radiation output of the CT system is expressed as the volume computed tomography dose index (CTDIvol), but also the tube charge (mAs) is provided for scans that are performed with an Aquilion 64 CT system (Toshiba Medical Systems, Japan) [34]. The clinically applied tube current, rotation time and pitch factor are associated with CTDIvols of $2.1 \mathrm{mGy}$ (newborn, $3.6 \mathrm{~kg}, 27 \mathrm{mAs}$ ), $2.9 \mathrm{mGy}$ (1 year old, $9.7 \mathrm{~kg}, 38 \mathrm{mAs}$ ), $4.1 \mathrm{mGy}$ (5 years old, $19.8 \mathrm{~kg}$, $53 \mathrm{mAs}$ ), $4.6 \mathrm{mGy}$ (10 years old, $33.2 \mathrm{~kg} .37 \mathrm{mAs})$, $7.1 \mathrm{mGy}$ (15 years old, $56.8 \mathrm{~kg}, 58 \mathrm{mAs}$ ), and $9.5 \mathrm{mGy}$ (adult, $74 \mathrm{~kg}, 77 \mathrm{mAs}$ ).

Dose calculations that were performed for the MIRD phantoms with the Monte Carlo software yielded organ doses, total body dose and effective dose according to ICRP 103. Dose assessment was performed for $12 \mathrm{CT}$ acquisitions, i.e. both for whole-body CT (including neck, chest and abdomen), and for CT of the neck and chest only, in all six age categories. These doses were incorporated into the risk model, as described under "Risk assessment". From these results, appropriate organ doses and the effective dose were derived for the year of diagnosis and treatment and for the following years of surveillance. If necessary, linear interpolation of dose values was performed to yield dose estimations at ages not included in the table.

\section{Radiation exposure from ${ }^{18} \mathrm{~F}$-FDG PET}

For assessment of organ dose and effective dose from ${ }^{18} \mathrm{~F}$ FDG PET, published tables were used that provide information about organ dose and effective dose per MBq of administered ${ }^{18}$ F-FDG activity. The ICRP provides information for 1-, 5-, 10- and 15-year-old children, and for adults [35]. Ruotsalainen et al. [36] published tables for estimation of radiation dose to the newborn in ${ }^{18} \mathrm{~F}$ FDG PET studies. For PET a dose of $3 \mathrm{MBq}$ of ${ }^{18} \mathrm{~F}-\mathrm{FDG}$ per kg body weight was assumed to be administered, based on the current state-of art imaging with integrated PET/CT systems (f.i. Biograph 40 TruePoint PET-CT, Siemens Medical Systems, Knoxville, TN, USA) . The five different age categories in the children being analysed in this study correspond to ${ }^{18} \mathrm{~F}-\mathrm{FDG}$ doses of $10 \mathrm{MBq}$ (newborn), $30 \mathrm{MBq}$ (1 year old), $60 \mathrm{MBq}$ (5 years old), $100 \mathrm{MBq}$ (10 years old) and $170 \mathrm{MBq}$ (15 years old). In adults, administration of $220 \mathrm{MBq}{ }^{18} \mathrm{~F}$-FDG was assumed.

\section{Risk assessment}

We performed risk assessment for five age categories of male and female paediatric patients diagnosed with HD (newborns, and children at the age of 1, 5, 10 and 15 years) and in three age categories for adult male and female patients diagnosed with DLBCL at the ages of 55, 65 and 75 years. 


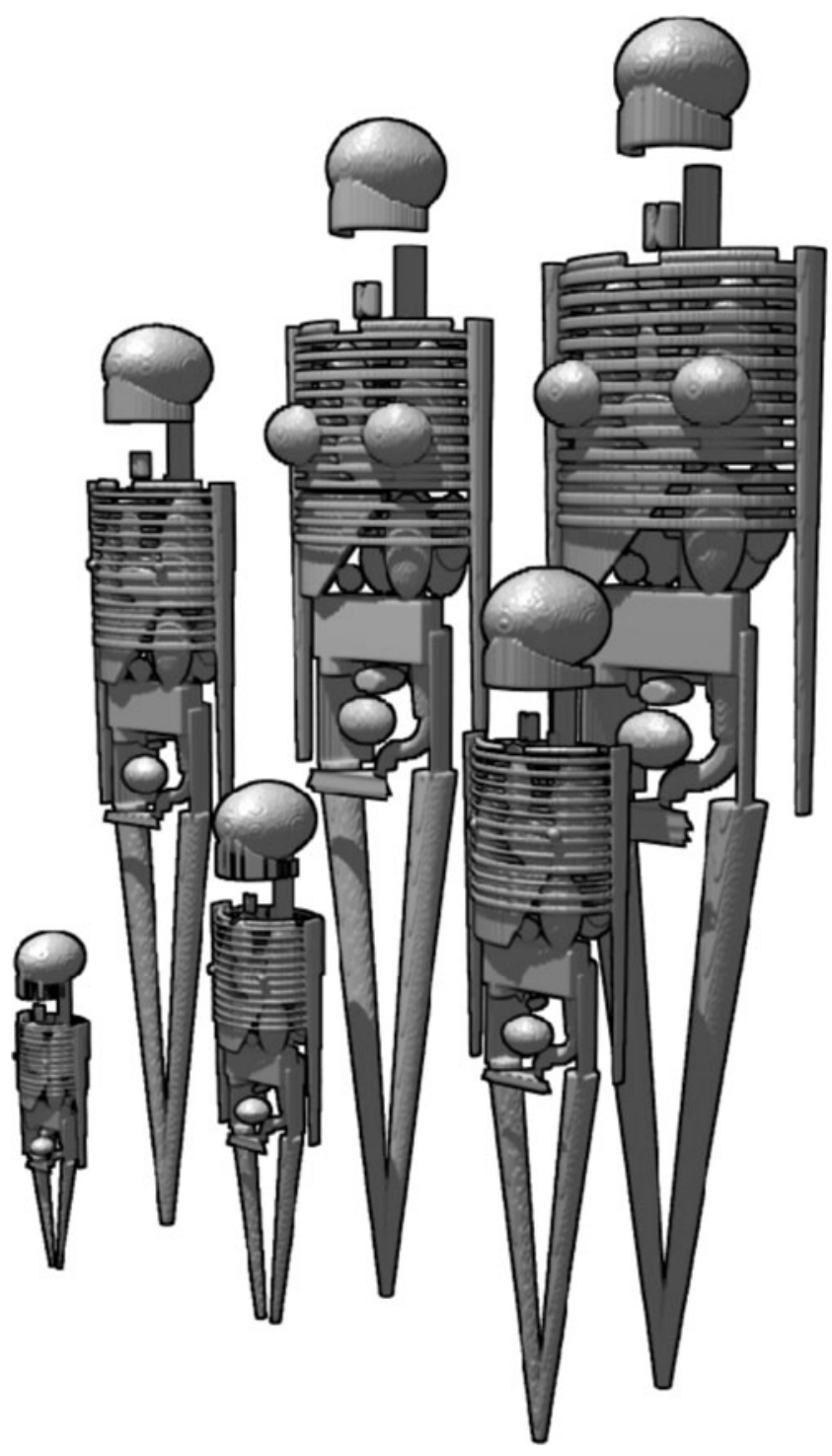

Fig. 1 The mathematical hermaphrodite Medical Internal Radiation Dose (MIRD) patient models; their body, skeleton and organs are described by spheres, ellipsoids and cones. The paediatric patient models are divided into five categories according to their age and weight: newborn (3.6 kg, left first row); 1 year old $(9.7 \mathrm{~kg}) ; 5$ years old $(19.8 \mathrm{~kg}) ; 10$ years old $(33.2 \mathrm{~kg}) ; 15$ years old $(56.8 \mathrm{~kg})$. The adult model represents an average-sized patient of $74 \mathrm{~kg}$ (right second row)

The BEIR VII excess relative risk (ERR) model was used for calculating radiation risk [37]. Chapter 12 of this BEIR report provides the equations and parameters for estimating organ-specific solid cancer mortality and leukaemia mortality. The ERR is expressed as a function of gender, absorbed organ dose, age at exposure and the attained age, and includes three organ-specific fit parameters. The organs are stomach, colon, liver, lung, breast, prostate, uterus, ovary, bladder, other organs and thyroid.

In the BEIR VII ERR model, the organ-specific excess risk for solid cancer mortality is expressed, relative to the gender- and age-dependent risk of the background cancer mortality for specific organs. This background is the naturally occurring mortality, not the mortality that is induced by radiation exposure during medical imaging. Data on the risk of naturally occurring cancer mortality depending on organ, gender and attained age were derived from ICRP Publication 103 (Euro-American cancer mortality rates by age and site) [38]. Subsequently, according to the BEIR VII model, an overall ERR function depending on organ dose, gender, age at exposure and attained age was calculated for male and female patients. Organ dose was incorporated into the risk model as a function of attained age; this was required because patients have different weights (and ages) and thus receive different associated radiation exposures during diagnosis, treatment and surveillance of malignant lymphoma. In addition to radiation risk, mortality rates that are typical for the young patient group with HD and the adult patient group with DLBCL could be taken into account, i.e. the overall 10-year survival for HD (94\%) and 5year survival for DLBCL (58\%) [27-30].

Risk calculations were performed using life tables, and they were done with and without taking into account the disease-related mortality. Life tables (also called mortality tables) are used in demography for measuring and modelling population processes. A life table shows, for each age, what the probability is that a person of that age will die before his or her next birthday. They allow for the calculation of the fraction of radiation-induced deaths, the reduction of life expectancy and the survival rate. An essential component of the life table is age- and gender-dependent mortality; in this study three different sources of mortality were integrated in the life tables: the background, the radiation induced, and the disease-related mortality. The background mortality that is typical for the asymptomatic population (gender- and age-specific probability of dying) was derived for the European population at large based on the Eurostat database [39]. The radiation-induced, age-, gender- and dose-dependent overall mortality that was calculated with the BEIR VII model as described in the previous section, was also incorporated into the life tables. Finally, disease-related mortality, as mentioned in the previous section, can also be integrated in the life tables. Calculations were carried out with the life tables with and without taking into account the mortality risks that are typical for patients with malignant lymphoma (HD- and DLBCL-related mortality rates).

\section{Results}

Radiation exposure from $\mathrm{CT}$ and ${ }^{18} \mathrm{~F}$-FDG PET

The radiation exposure from whole-body $\mathrm{CT}$, from $\mathrm{CT}$ of the neck and thorax, and from whole-body ${ }^{18} \mathrm{~F}$-FDG PET 


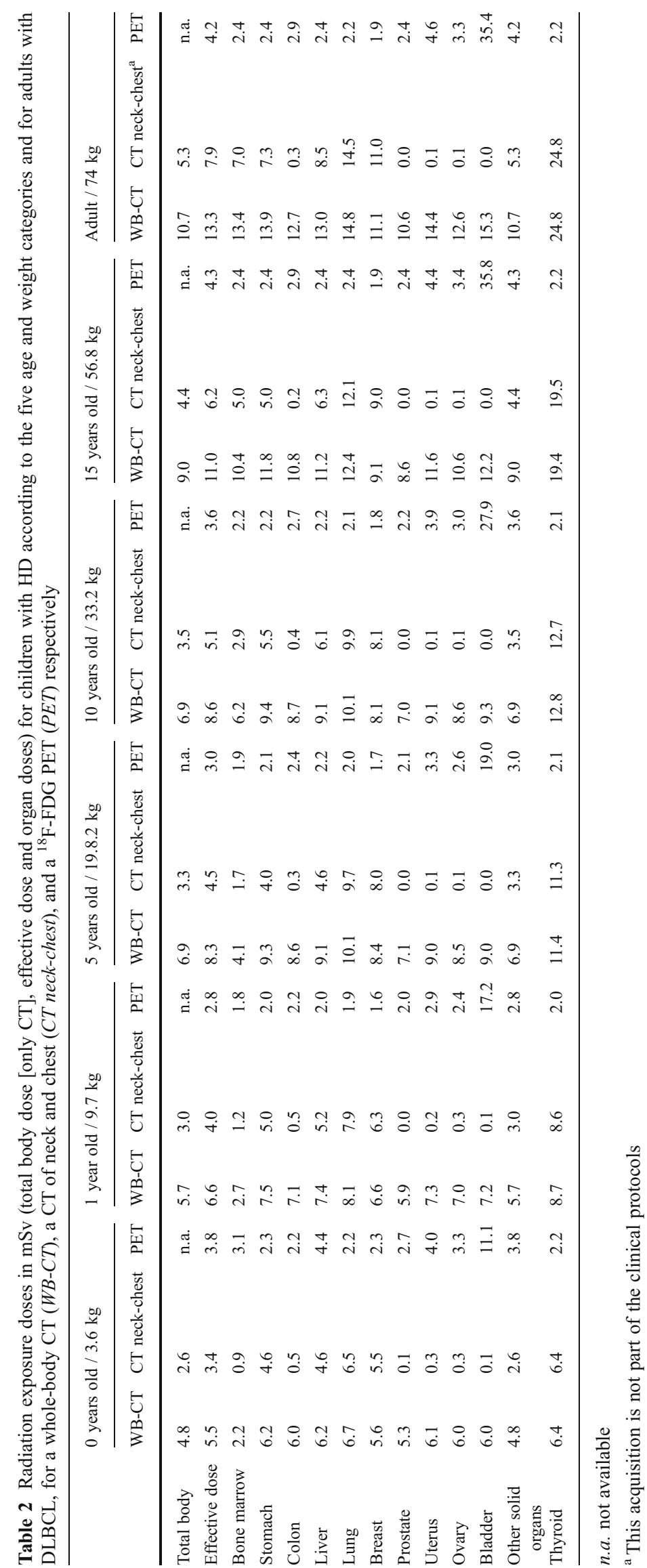


for children (HD) according to the five age categories and for adults (DLBCL) are presented in Table 2. Effective dose increased gradually with age from $5.5 \mathrm{mSv}$ to $13.3 \mathrm{mSv}$ (whole-body CT), and from $3.4 \mathrm{mSv}$ to $7.9 \mathrm{mSv}$ (neck-chest CT). For ${ }^{18}$ F-FDG PET examinations the variations in effective dose were smaller, ranging from $2.8 \mathrm{mSv}$ to $4.3 \mathrm{mSv}$. In CT the highest organ doses were observed for lung and thyroid, and variations in organ dose were modest for organs lying entirely within the anatomical area examined. In PET the highest organ dose by far was observed for the bladder.

Neonates and children with HD usually undergo seven whole-body CTs, five of the neck and chest, and two ${ }^{18} \mathrm{~F}$ FDG PETs over a period of 5 years. Adults with DLBCL usually undergo seven whole-body CTs and one ${ }^{18} \mathrm{~F}-\mathrm{FDG}$ PET during a period of 2.5 years. Because of the intensive use of X-ray imaging, the effective dose accumulates rapidly in patients with malignant lymphoma. In children with HD, cumulative effective dose reaches $66 \mathrm{mSv}$ (newborn at diagnosis), $69 \mathrm{mSv}$ (1 year old at diagnosis), $80 \mathrm{mSv}$ (5 years old at diagnosis), $90 \mathrm{mSv}$ (10 years old at diagnosis) and $113 \mathrm{mSv}$ (15 years old at diagnosis). Differences between these paediatric age categories are due to differences in patient size and the patient size-specific acquisition protocols for both $\mathrm{CT}$ and ${ }^{18} \mathrm{~F}$-FDG PET. In adults with DLBCL the cumulative effective dose reaches $97 \mathrm{mSv}$ 2.5 years after diagnosis.

\section{Risk assessment}

Demographic and risk-related parameters were derived from the life tables for neonates and children (with HD) corresponding to the five different age and weight categories. The paediatric categories are identified by the age at diagnosis (Table 3) and for adult patients (with DLBCL) the categories were 55, 65 and 75 years of age at diagnosis of the disease (Table 4).

Calculations were performed with and without taking into account the disease-related mortality rates typical for the patient cohorts (Table 3). Risk assessment taking into account the effects of disease-related mortality resulted in a substantially lower fraction of radiation-induced deaths, and a lower associated reduction of life expectancy.

The calculations, which included disease-related mortality [or without disease-related mortality in brackets], resulted in an average 10-year survival of 94\% (range 9394\%) $[100 \%$ (range $99-100 \%$ )] for the young patient group with HD and an average 5-year survival of 55\% (range 46$59 \%$ ) [90\% (range 76-98\%)] for the adult patient group with DLBCL. Within the paediatric patient group, the average fraction of radiation-induced deaths was $0.4 \%[0.6 \%]$ for boys and $0.7 \%[1.1 \%]$ for girls. Within the adult group these values were even smaller; $0.07 \%[0.28 \%]$ for men and $0.09 \%[0.37 \%]$ for women. The average radiation-induced

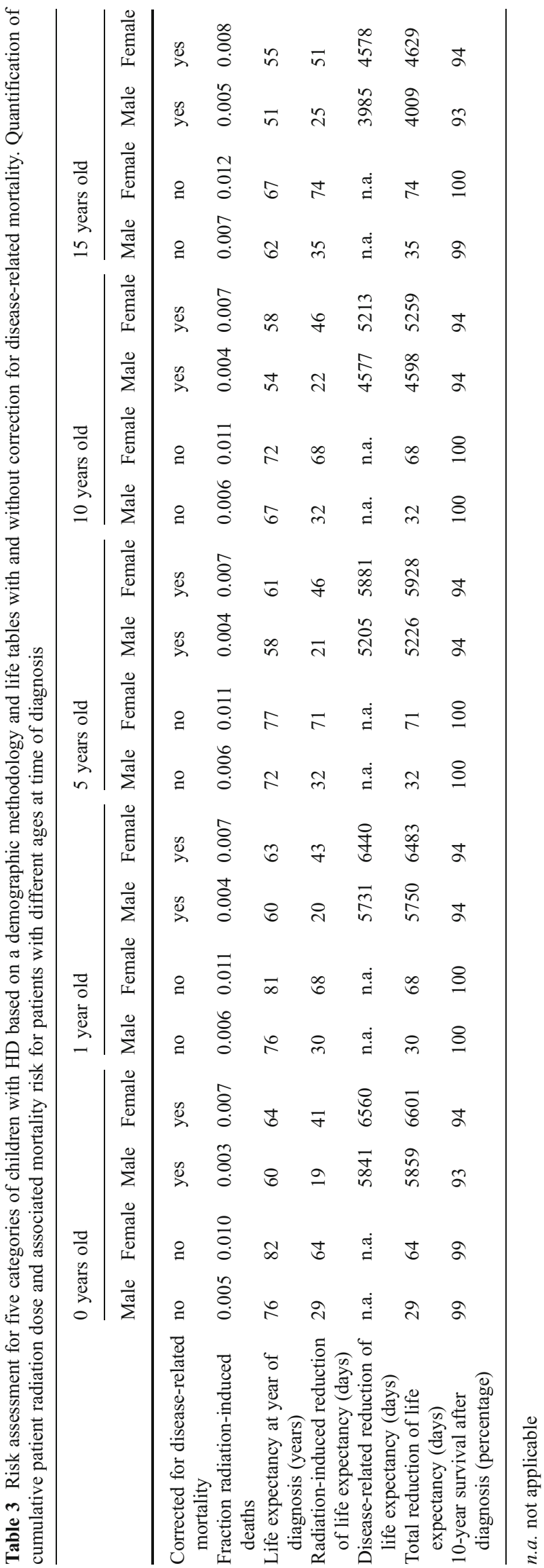


Table 4 Risk assessment for three categories of adults with nonHodgkin's lymphoma (NHL), subtype DLBCL, based on a demographic methodology and life tables with and without correction for disease-related mortality. Quantification of cumulative patient radiation dose and associated mortality risk for patients with different ages at time of diagnosis

\begin{tabular}{|c|c|c|c|c|c|c|c|c|c|c|c|c|}
\hline & \multicolumn{4}{|c|}{55 years old } & \multicolumn{4}{|c|}{65 years old } & \multicolumn{4}{|c|}{75 years old } \\
\hline & Male & Female & Male & Female & Male & Female & Male & Female & Male & Female & Male & Female \\
\hline Corrected for disease-related mortality & no & no & yes & yes & no & no & yes & yes & no & no & yes & yes \\
\hline Fraction of radiation-induced deaths & 0.004 & 0.005 & 0.001 & 0.001 & 0.003 & 0.004 & 0.001 & 0.001 & 0.002 & 0.002 & 0.001 & 0.001 \\
\hline Life expectancy at year of diagnosis (year) & 25 & 29 & 9 & 9 & 17 & 20 & 7 & 8 & 10 & 12 & 6 & 6 \\
\hline $\begin{array}{l}\text { Radiation-induced reduction of life expectancy } \\
\text { (days) }\end{array}$ & 14 & 23 & 2 & 2 & 9 & 13 & 2 & 2 & 4 & 5 & 1 & 1 \\
\hline $\begin{array}{l}\text { Disease-related reduction of life expectancy } \\
\text { (days) }\end{array}$ & n.a. & n.a. & 5931 & 7228 & n.a. & n.a. & 3486 & 4354 & n.a. & n.a. & 1715 & 2057 \\
\hline Total reduction of life expectancy (days) & 14 & 23 & 5933 & 7230 & 9 & 13 & 3488 & 4356 & 4 & 5 & 1716 & 2058 \\
\hline 5-year survival after diagnosis (percentage) & 96 & 98 & 58 & 59 & 90 & 95 & 55 & 58 & 76 & 86 & 46 & 52 \\
\hline
\end{tabular}

n.a. not applicable

reduction of life expectancy in paediatric patients with HD is 21 days for boys and 45 days for girls. In adults with DLBCL, the average radiation-induced reduction of life expectancy is 1.5 days in men and 2.0 days in women.

\section{Discussion}

This study shows that the effect of disease-related mortality on radiation risk assessment in patients with malignant lymphoma is substantial. By taking into account diseaserelated mortality, the proportion of radiation-induced deaths decreased between $30 \%$ and $40 \%$ in paediatric patients with $\mathrm{HD}$, and between $50 \%$ and $80 \%$ in adults with DLBCL. Radiation-induced reduction of life expectancy decreased by similar percentages. Our methodology, which integrates a model for organ dose assessment and risk assessment in one demographic model, showed that the potential radiation-induced reduction of life expectancy is only a small fraction compared with the disease-related reduction of life expectancy, namely a fraction between 0.003 and 0.011 for HD (children); and between 0.0003 and 0.0006 for DLBCL (adults). This reflects the fact that the radiationrelated risk is a late risk with its expression up to decades after the actual exposure and has therefore less effect on the reduction of life expectancy. Our methodology for radiation risk assessment is an improvement compared with the usual standard of practice, where disease-related mortality is not considered. Although the observed cumulative effective doses are relatively high for imaging patients with malignant lymphoma, the associated estimated radiation risks are still very modest.

Within the paediatric patient group, it is estimated that, on average, $0.4 \%$ of male and $0.7 \%$ of female patients eventually die because of the radiation exposure associated with medical imaging. For adult patients diagnosed with DLBCL, it is estimated that $0.07 \%$ of male and $0.09 \%$ of female patients die because of radiation exposure. The higher values for women result mainly from the relatively high sensitivity of the female breast to radiation exposure [40].

Calculation in which the disease-related mortality was taken into account resulted in an average 10-year survival of $94 \%$ (range, 93-94\%) for the young patient group with HD, which matches excellently with the published overall 10 -year survival of $94 \%[27,28]$. Similarly, for the adult patient group with DLBCL, the calculated average 5-year survival was $55 \%$ (range, 46-59\%), which matched well with the published value of $58 \%[29,30]$. The good agreement between our calculated reduction in survival after diagnosis and published values is a clear indication that risk assessment should be performed taking disease-related mortality into account.

This study has some limitations. Malignant lymphoma comprises a heterogeneous group of entities, with more than 40 subtypes [41]. In addition, with regard to radiation exposure, age is an important factor. Therefore we chose to analyse the risk of radiation exposure in only two types of lymphoma, HD in paediatric patients and DLBCL in adult patients (the most common malignant lymphoma subtypes in these age groups). Another limitation is that the assumed 10- and 5-year survival rates (for HD and DLBCL, respectively) may be an underestimation of the current situation, because treatment strategies have continued to evolve [27-30]. In this study, we focused on the mortality risk caused by the radiation exposure of the patient. The morbidity risk induced by the radiation exposure was not considered. It can be assumed that not all patients with a radiation-induced malignancy die from this malignancy, and more patients suffer from radiation-induced diseases 
than the number of patients expressed in the mortality risk. Our calculations are based on a methodology that is according to broadly accepted dosimetric techniques and risk models. However, radiation dose assessment and risk assessment at the low dose levels that are common in diagnostic radiology are always associated with considerable uncertainties, implying that the absolute risk figures that we calculated should be interpreted with care.

Furthermore, with the implementation of integrated PETCT systems, low-dose CT is performed in addition to PET, as it is used for attenuation correction in PET imaging. The use of CT for attenuation correction reduces the examination time, which implies a lower dose of FDG $(5 \mathrm{MBq} / \mathrm{kg}$ vs $3 \mathrm{MBq} / \mathrm{kg}$ ). The low-dose CT was not included in this study. Two or one additional low-dose CT(s) would have been performed in children with HD and in adults with DLBCL, respectively, with each low-dose CT causing extra radiation exposure of only approximately $3 \mathrm{mSv}$ for adults [42]. Finally, it should be recognised that there are considerable uncertainties in the radiation risk model of BEIR, especially regarding the risk at low-dose levels such as those encountered in CT [17].

In order to establish the most efficient imaging strategy and limit the radiation exposure in patients with malignant lymphoma, there are different options. It may be sufficient to use only the low-dose whole-body CT (combined with FDG-PET) during and after therapy instead of a diagnostic (i.e. full-dose) whole body CT. This implies a reduction in radiation exposure of 6 times $8 \mathrm{mSv}$ for adults. In patients with a FDG-avid type of malignant lymphoma and a baseline ${ }^{18} \mathrm{~F}$-FDG PET-CT combined with a diagnostic $\mathrm{CT}$, the most accurate and appropriate method of imaging during follow-up may therefore prove to be the low dose PET-CT, without the diagnostic CT. Another recent development is the introduction of whole-body magnetic resonance imaging (MRI) for the evaluation of malignant lymphoma. Initial results on this application of wholebody MRI are promising, but more research is still needed before it can be recommended as an alternative to CT and/or ${ }^{18}$ F-FDG PET [43-46].

In conclusion, the disease-related reduction in life expectancy of patients diagnosed with malignant lymphoma must be taken into account to achieve more realistic estimates of radiation risk. It results in higher overall mortality and substantial lower incidence of radiation-induced deaths. Although the cumulative effective dose from medical imaging is high, the actual calculated radiation risks are very modest. The radiation exposure that results from imaging with $\mathrm{CT}$ and ${ }^{18} \mathrm{~F}$-FDG PET is considered as justified in patients with malignant lymphoma, but should still be performed with care, especially in children. Ongoing studies have to establish the most efficient imaging strategies for (the different subtypes of) malignant lymphoma.
Open Access This article is distributed under the terms of the Creative Commons Attribution License which permits any use, distribution, and reproduction in any medium, provided the original author (s) and the source are credited.

\section{References}

1. Kwee TC, Kwee RM, Nievelstein RA (2008) Imaging in staging of malignant lymphoma: a systematic review. Blood 111:504-516

2. Hoskin PJ (2003) PET in lymphoma: what are the oncologist's needs? Eur J Nucl Med Mol Imaging Supply 1:S37-S41

3. Carbone PP, Kaplan HS, Musshoff K, Smithers DW, Tubiana M (1971) Report of the committee on Hodgkin's disease staging classification. Cancer Res 11:1860-1861

4. Armitage JO (2005) Staging non-Hodgkin lymphoma. CA Cancer $\mathrm{J}$ Clin 55:368-376

5. Hoh CK, Glaspy J, Rosen P et al (1997) Whole-body FDG-PET imaging for staging of Hodgkin's disease and lymphoma. J Nucl Med 38:343-348

6. Buchmann I, Reinhardt M, Elsner K et al (2001) 2-(fluorine-18) fluoro-2-deoxy-D-glucose positron emission tomography in the detection and staging of malignant lymphoma. A bicenter trial. Cancer 91:889-899

7. Seam P, Juweid ME, Cheson BD (2007) The role of FDG-PET scans in patients with lymphoma. Blood 110:3507-3516

8. Juweid ME, Stroobants S, Hoekstra OS et al (2007) Use of positron emission tomography for response assessment of lymphoma: consensus of the imaging subcommittee of international harmonization project in lymphoma. J Clin Oncol 25:571-578

9. Cheson BD, Pfistner B, Juweid ME et al (2007) Revised response criteria for malignant lymphoma. J Clin Oncol 25:579-586

10. Brenner D, Elliston C, Hall E, Berdon W (2001) Estimated risks of radiation-induced fatal cancer from pediatric CT. AJR Am J Roentgenol 176:289-296

11. Brenner DJ, Elliston CD (2004) Estimated radiation risks potentially associated with full-body CT screening. Radiology 232:735738

12. Linton OW, Mettler FA Jr (2003) National conference on dose reduction in CT, with an emphasis on pediatric patients. AJR Am J Roentgenol 181:321-329

13. Executive Summary Board on Radiation Effects ResearchDivision on Earth and Life Studies (2005) Health risks from exposure to low levels of ionizing radiation: BEIR VII-Phase 2. National Academy Press, Washington

14. Preston DL, Shimizu Y, Pierce DA, Suyama A, Mabuchi K (2003) Studies of mortality of atomic bomb survivors. Report 13: solid cancer and noncancer disease mortality: 1950-1997. Radiat Res 160:381-407

15. Pierce DA, Preston DL (2000) Radiation-related cancer risks at low doses among atomic bomb survivors. Radiat Res 154:178-186

16. Preston DL, Ron E, Tokuoka S et al (2007) Solid cancer incidence in atomic bomb survivors: 1958-1998. Radiat Res 168:1-64

17. National Council on Radiation Protection and Measurements (1997) Uncertainties in fatal cancer risk estimates used in radiation protection. Report 126. NCRP, Bethesda

18. Amis ES Jr, Butler PF, Applegate KE et al (2007) American college of radiology white paper on radiation dose in medicine. $\mathrm{J}$ Am Coll Radiol 4:272-284

19. Hall EJ, Brenner DJ (2008) Cancer risks from diagnostic radiology. Br J Radiol 81:362-378

20. Hillman BJ (2008) Radiation exposure and imaging utilization. J Am Coll Radiol 5:689-690

21. Mezrich R (2008) Are CT scans carcinogenic? J Am Coll Radiol 5:691-693 
22. Mettler FA Jr, Thomadsen BR, Bhargavan M et al (2008) Medical radiation exposure in the U.S. in 2006: preliminary results. Health Phys 95:502-507

23. Connors JM (2005) State-of-the-art therapeutics: Hodgkin's lymphoma. J Clin Oncol 23:6400-6408

24. Ansell SM, Armitage J (2005) Non-Hodgkin lymphoma: diagnosis and treatment. Mayo Clin Proc 80:1087-1097

25. Hennessy BT, Hanrahan EO, Daly PA (2004) Non-Hodgkin lymphoma: an update. Lancet Oncol 5:341-353

26. Brenner DJ, Shuryak I, Einstein AJ (2011) Impact of reduced patient life expectancy on potential cancer risks from radiologic imaging. Radiology 261:193-198

27. von der Weid NX (2008) Adult life after surviving lymphoma in childhood. Support Care Cancer 16:339-345

28. Chow LM, Nathan PC, Hodgson DC et al (2006) Survival and late effects in children with Hodgkin's lymphoma treated with MOPP/ ABV and low-dose, extended-field irradiation. J Clin Oncol 24:5735-5741

29. Illidge T, Tolan $S$ (2008) Current treatment approaches for diffuse large B-cell lymphoma. Leuk Lymphoma 49:663-676

30. Feugier P, Van HA, Sebban C et al (2005) Long-term results of the R-CHOP study in the treatment of elderly patients with diffuse large B-cell lymphoma: a study by the Groupe d'Etude des Lymphomes de l'Adulte. J Clin Oncol 23:4117-4126

31. Cristy M (1980) Mathematical phantoms representing children of various ages for use in estimates of internal dose. U.S. Nuclear Regulatory Commission Rep. NUREG/CR-1159 (also Oak Ridge National Laboratory Rep. ORNL/NUREG/TM-367)

32. Deak P, van Straten SM, Shrimpton PC, Zankl M, Kalender WA (2008) Validation of a Monte Carlo tool for patient-specific dose simulations in multi-slice computed tomography. Eur Radiol 18:759-772

33. Nievelstein RA, van Dam IM, van der Molen AJ (2010) Multidetector CT in children: current concepts and dose reduction strategies. Pediatr Radiol 40:1324-1344

34. Nitt-Gray MF (2002) AAPM/RSNA physics tutorial for residents: topics in CT. Radiation dose in CT. Radiographics 22:1541-1553
35. Valentin DJ (1998) Radiation dose to patients from radiopharmaceuticals (Addendum 2 to ICRP Publication 53). Ann ICRP 28:1

36. Ruotsalainen U, Suhonen-Polvi H, Eronen E et al (1996) Estimated radiation dose to the newborn in FDG-PET studies. J Nucl Med 37:387-393

37. NAS/NRC (2006) Health risks from exposure to low levels of ionising radiation: BEIR VII phase 2. Board on Radiation Effects Research National Research Council of the National Academies, Washington

38. The 2007 Recommendations of the International Commission on Radiological Protection. ICRP publication 103. Ann ICRP 37:1332

39. Eurostat (2007) http://epp.eurostat.ec.europa.eu/

40. De Bruin ML, Sparidans J, van't Veer MB et al (2009) Breast cancer risk in female survivors of Hodgkin's lymphoma: lower risk after smaller radiation volumes. J Clin Oncol 27:4239-4246

41. Swerdlow S, Campo E, Harris N (2008) WHO classification of tumours of haematopoietic and lymphoid tissues, 4th edn. International Agency for Research on Cancer, Lyon

42. Brix G, Lechel U, Glatting G et al (2005) Radiation exposure of patients undergoing whole-body dual-modality 18 F-FDG PET/CT examinations. J Nucl Med 46:608-613

43. Kellenberger CJ, Miller SF, Khan M, Gilday DL, Weitzman S, Babyn PS (2004) Initial experience with FSE STIR whole-body MR imaging for staging lymphoma in children. Eur Radiol 14:1829-1841

44. Brennan DD, Gleeson T, Coate LE, Cronin C, Carney D, Eustace SJ (2005) A comparison of whole-body MRI and CT for the staging of lymphoma. AJR Am J Roentgenol 185:711-716

45. Kwee TC, Quarles van Ufford HM, Beek FJ et al (2009) Wholebody MRI, including diffusion-weighted imaging, for the initial staging of malignant lymphoma: comparison to computed tomography. Invest Radiol 44:683-690

46. Quarles van Ufford HM, Kwee TC, Beek FJ et al (2011) Newly diagnosed lymphoma: initial results with whole-body T1weighted, STIR, and diffusion-weighted MRI compared with 18 F-FDG PET/CT. AJR Am J Roentgenol 196:662-669 